\title{
Comparing the Bulk Modulus of Aerogels Obtained Through Different Formulas
}

\author{
Ryan Aycock* \\ Department of Emergency Medicine, USA \\ *Corresponding author: Ryan Aycock, Department of Emergency Medicine, USA

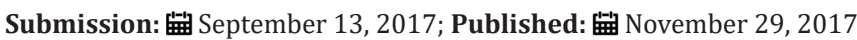

\begin{abstract}
Aerogels are highly porous silica networks which lend themselves to several unique properties including low densities, small dielectric constants, and high impact absorption. While this list of attributes is impressive, these materials are extremely fragile. By using organic polymers as additives, researchers have discovered that gels can be made tougher while retaining many of their unique features. This paper compares the bulk moduli of pure silica aerogels to those containing collodion (nitro cellulose) by employing the novel technique of mercury porosimetry.
\end{abstract}

\section{Introduction}

\section{Highlights of aerogels}

Nicknamed "solid smoke" by their observers, aerogels has gained a lot of attention due to their unique properties [1]. These light weight materials are widely studied for their thermoinsulative and kinetic behaviors and currently hold the top spot in 14 categories in The Guinness Book of World Records.

Aerogels are highly porous materials which are generally made from silica networks. Generally comprised of $80 \%$ or more air, they have dielectric values as low as 1.4 [2]. Joel Plawsky of Rensselaer Polytechnic Institute predicts that insulators fabricated from aerogels may single handedly double computer speeds. Thin films of aerogels have been examined for a variety of uses including interlayer dielectrics [3]. When NASA launched the Sojourner Mars rover in 1997, the agency employed aerogels to protect the vehicle's sensitive electronics from the harsh, freezing environment of the Martian surface [4]. Heather Paul of the Johnson Space Center has further suggested that an aerogel void medium could be bound by fibers and used in space suits for future manned missions to Mars [5].

In the civilian sector, Cabot Corp. has developed a way to create aerogels in a bulk continuous process [6,7]. Calling the material nanogel, the company is already installing window panes made from gels in the roofs of motel swimming pools. The basis of their product is a flexible aerogel blanket enclosed in fibers [8]. In addition, scientists are hoping that this super light insulating substance can be used in fabrics and winter clothing. Improvements in cost could also allow companies such as Maytag and General Electric to create more energy efficient home appliances.
While aerogels are indeed very brittle, they can absorb a considerable amount of energy [9]. Upon impact, the silica network of aerogels collapses, releasing the gases contained within. Given that this substance has very small pores in the angstrom to nanometer size range air must slowly pass through the capillaries as the network gives way, spreading the impact over a longer period of time. Also, given that most aerogels are completely inorganic, they do not suffer the rebound effect demonstrated in many organic types of foam. This recoil produced in carbon based materials transfers' energy back into the impact object and may cause damage to the protected entity. For this reason, NASA decided to employ aerogels in its star dust spacecraft [10]. The ongoing purpose of the exploratory mission is to catch particles in the wild 2 comet. Traveling at speeds of $136,000 \mathrm{mph}$, the comet's dust would easily vaporize upon impact or become distorted if another material were used [4].

Further, aerogels have been adapted into Cerenkov radiation detectors for high energy physics [11]. With continued development, automobile manufactures could use gels to reduce the damage caused by car accidents. The collapsible silica network might well be one of many advances in improving the safety of vehicles.

The list of applications above represents some of the secondary properties of aerogels. It is their porosity which lends their amazing thermal and kinetic behavior. While these uses are impressive and can certainly be important in future industrial and commercial expenditures, the medical establishment could profit greatly from the pores themselves. El Rassy [12] discovered that enzymes could be encapsulated into the aerogel network and that the substrates' diffusion could be homogeneously dispersed. In addition, he 
demonstrated that proteins such as lipase show increased catalytic effects when introduced into aerogels. The pores do such a wonderful job in retaining particles that one author called aerogels a "nanoglue" [13]. When composed of iron, boron and neodymium, aerogels retain magnetic orientations, making them very attractive in permanent magnetic systems [14]. Physicists in Spain hope that these transparent gels could eventually be incorporated into magneto-optical memory devices. For a list of more aerogel uses, see [15].

\section{Making an aerogel}

Aerogels such as those used in the studies listed above typically are created from tetramethoxysilane (TMOS). Since TMOS is very ex- pensive and extremely toxic, tetraethoxysilane (TEOS) may be used for many research and "skunk works" projects because it is cheaper and less hamful [16].

To create a silica gel network, TMOS must first be hydrolyzed as shown below:

$$
\begin{gathered}
\mathrm{Si}\left(\mathrm{OCH}_{3}\right)_{4}+\mathrm{H}_{2} \mathrm{O} \rightleftharpoons \mathrm{Si}\left(\mathrm{OCH}_{3}\right)_{3}(\mathrm{OH})+\mathrm{CH}_{3} \mathrm{OH} \\
\mathrm{Si}\left(\mathrm{OCH}_{3}\right)_{3}(\mathrm{OH})+\mathrm{Si}\left(\mathrm{OCH}_{3}\right)_{3}(\mathrm{OH}) \rightleftharpoons\left(\mathrm{H}_{3} \mathrm{CO}\right)_{3} \mathrm{SiOSi}\left(\mathrm{OCH}_{3}\right)_{3}+\mathrm{H}_{2} \mathrm{O}
\end{gathered}
$$

The reaction continues to polymerize.

Generally, an acid or base must used as a catalyst. The TMOS forms silica colloidal particles which become interconnected with each other by silica polymers [12]. Acid catalysts such as $\mathrm{HF}$ or $\mathrm{HCl}$ create linear chains and a low density of cross-linking networks. Gels created with this method shrink easily during drying an important property we will observe later. Base catalysis, on the other hand, yields a high density of cross links and enhances polymerization [17]. After hydrolysis, the solution must then undergo condensation. This second step may be enhanced with another catalyst such as NH3 [18], but such an additive is not necessary.

While aerogels are typically made from silica, they can be created from organic compounds such as poly (vinyl chloride) [19]. Further, mixtures of TMOS and carbon based materials can lead to gels which are still light weight, yet are much stronger and tougher.

Being comprised of only glass and air, aerogels are extremely fragile just holding one can cause fracture. In order to lead towards a wide-spread commercial adoption of aerogels, these products must be strengthened either through densification or through a change in formulation. By introducing a polymeric phase, Premachandra [11] claims that we can decrease the brittleness of the ceramic phase. Similarly, Nicholas Leventis describes a method in which a polyurethane additive was used to link silica particles, producing aerogels which are just as light as before, yet over 100 times stronger [20]. He goes so far as to liken the hybrid substance created from his method as a new kind of fiberglass [21].

This paper will examine the differences in mechanical properties obtained from two recipes. The first set of aerogels will be a simple silica based sol gel, produced according to Kevin \& Powers [16] formulation. The second collection will contain a nitro cellulose additive. The goal of this endeavor is to create stronger aerogels without sacrificing their light weight feature. The bulk modulus, a measure of a material's compressability, will quantify this project's efforts as this method has been used to gauge the strength of gels in the past [22]. The modulus will be determined using a mercury porosimeter.

\section{Using a mercury porosimeter to measure mechanical properties}

While some authors have managed to test elastic properties in three-point flexure [21] and AFM [22], simply placing a pure silica aerogel in such an instrument is enough of a disturbance to cause a fracture. Further, a scan of current literature reveals that no one has ever tested the hardness. So delicate are these gels that in some size ranges, one researcher concludes, "There is no reliable, proven method to measure E [Young's modulus] on soft thin films" [23]. He states that while mechanical properties of aerogels are important, they are difficult to quantify. His claim leads us to realize that when traditional methods fail, we must consider novel approaches.

While this method is typically used to determine pore size [24], we can determine various mechanical properties. According to Majling [25], mercury will not enter the pores of aerogels. Instead, the gels are isostatically compressed according to the "knee model" [26]. This configuration states that the porous network of aerogels can be considered as a bar like structure, where the legs bend like a knee under the application of uniaxil stress. The restoring forces become weaker with decreasing angles between the legs, leading to weaker mechanical behavior [27]. The decrease in modulus cannot be seen as micro-cracks, however, but rather are the rupture of chains connecting near neighbors [26].

Instead of completely sintering in a porosimeter as once expected [28], these gels yield a volume/pressure plot comprised of two portions: a linear beginning followed by a deviation an indication that increased pressures does lead to a stronger structure [25]. The linear portion can be used to calculate the bulk modulus in the elastic range according to the equation [29]: Majling [25] proposes that mercury porosimeters can evaluate mechanical behavior of aerogels. In this technique, mercury is forced under pressure into a capillary until the external force is balanced by the surface tension.$$
K=V \frac{d P}{d V}
$$ \\ Materials and Methods \\ Forming a characteristic contact angle [30] (See Figure 1). For this experiment, I tested the two recipes for aerogels given below.}

\section{Formulation (a)}

Beginning with an ice bath to cool the in- gredients to $4{ }^{\circ} \mathrm{C}$, I mixed $50 \mathrm{~mL}$ water (purified through a Nanopure Infinity from Barn- stead), 50mL methanol (CAS 67-56-1, Fisher Scientific \#A452-4), 10mL 3\% HF (derived from 49\% HF, CAS 7664-39-3, Fisher Scientific \#A147-1), and 4mL 1N $\mathrm{HNO}_{3}$ (CAS 7697-37-2, 
Fisher Scientific, \#A200) in a Teflon con- tainer and left it on a stir plate until the mixture had cooled. I then added $35 \mathrm{~mL}$ TEOS (CAS 78-10-4, Acros Organics, \#157810010) and let the solution continue stirring for an addition 5 minutes. I then removed the sample and divided it into $20 \mathrm{~mL}$ parts and placed each fraction into polymethylpentane (PMP) containers (Nalgene) and tightly capped the canister. PMP is a very smooth, very hydrophobic polymer which is not attacked by TEOS and keeps the gel intact while it ages [26].

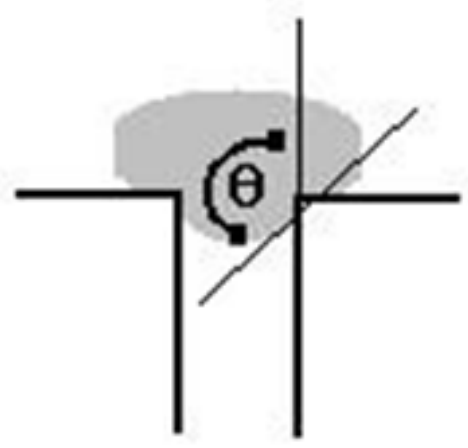

Figure 1: A mercury drop resists entering the capillary, bulging only a little way into the pas- sage until its weight is balanced by the surface tension.

After letting the solution rest for one hour, a gel formed. I subsequently opened the containers and soaked them in excess dry methanol for 12 hours to solvent exchange out the water. I repeated this step two additional times with fresh batches of dry methanol, again at 12 hours apiece. I then placed a single canister in a super critical drier (SPI \#13200J) and solvent exchanged the methanol with liquid $\mathrm{CO}_{2}$ three times at 2 hours each occasion.

Finally, I heated the $\mathrm{CO}_{2}$ to $40{ }^{\circ} \mathrm{C}$ which raised the pressure to 1300 psi. At this critical point, I slowly exhausted the gas over the course of an additional 2 hours. Because super critical drying

\section{Result}

preserves the gel structure and prevents cracking from the buildup of capillary forces [13], the result was a light blue aerogel comprised of nearly $80 \%$ air.

\section{Formulation (b)}

For this second recipe, I wanted to use nitrocellulose (collodion) as an organic stabilizer to test the predictions that polymers lead to increased strength. Since cellulose precipitates upon the addition of water, this formulation contains no water. Given that an acid catalyst could also cause precipitation, a neutral catalyst was needed. I mixed 50mL acetone (CAS 67-64-1, Fisher Scientific \#A949-4), 10mL collodion (CAS 9004-70-0, Fisher Scientific \#UN 2059), and 10mL $0.01 \mathrm{M}$ NH4F in a Teflon container. The result was an endothermic reaction with a neutral $\mathrm{pH}$. I then added $35 \mathrm{~mL}$ TEOS and stirred the mixture for 5 minutes. Just as in the first formulation, I separated the solution into $20 \mathrm{~mL}$ parts and placed the portions in PMP canisters. This time, however, the ingredients took an average of two weeks to gel as opposed to 1 hour as observed with formulation A. The absence of water is one possible cause for the delay. Further, the gel was a white solid rather than the colorless variety as experienced earlier meaning that cellulose had slowly precipitated during gelation. I performed a liquid $\mathrm{CO}_{2}$ solvent exchange followed by supercritical drying as outlined under the first formulation. The outcome was a light, yet flaky solid. Upon initial glance, this second set of aerogels did appear to be stronger than the first batch as the overall structure held together more readily.

\section{The porosimeter}

Two gels from each batch were selected for the porosimeter test. They were broken into subcubic centimeter sized fragments and outgassed at $110{ }^{\circ} \mathrm{C}$ for no less than 1 hour. All trials were performed on an Autoscan 60 Mercury Porosimeter (Quanta Chrome \#05014). All runs assumed a contact angle of $140{ }^{\circ} \mathrm{C}$.

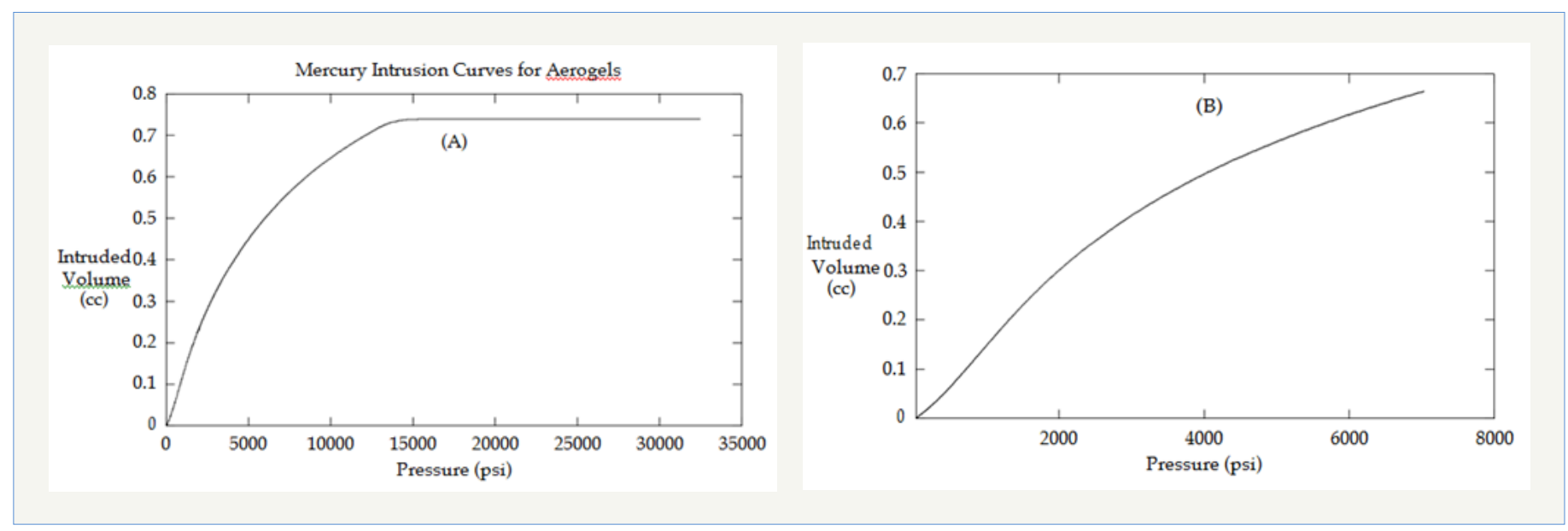



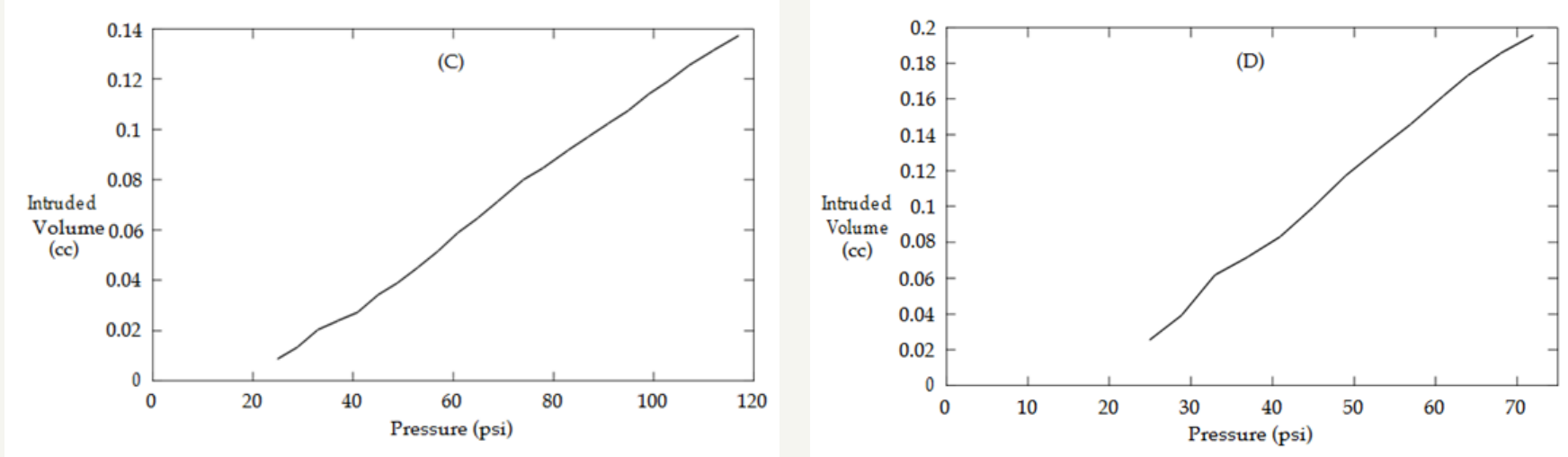

Figure 2: Mercury intrusion curves for the four tested gels. (A) and (B) are pure silica aerogels.

(C) and (D) contain collodion.

Shown in Figure 2 (spanning pages 5-6) are the volume/ pressure curves given by the mercury porosimeter. All gels have linear beginnings (see Figure 3 for a close up of gel B). Gels A and B demonstrate expected results [16] while $C$ and $D$ do not. In fact, the porosimeter halted around 100 psi for these two gels. This outcome is much unexpected and demonstrates that not all organic polymers lead to stronger aerogels.

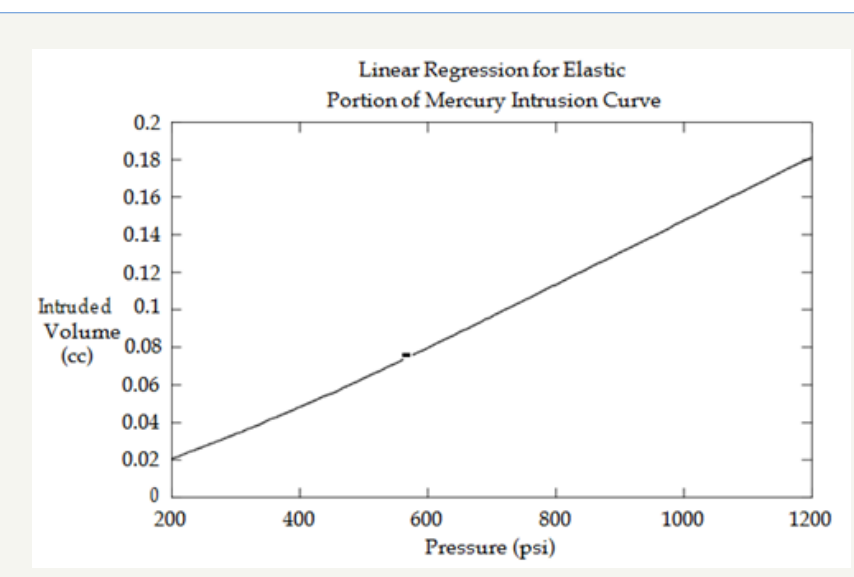

Figure 3: Close-up of the linear portion from Figure 2 (B). In this example, regression analysis gives a slope of $1.6 \times 10^{-4} \mathrm{cc} / \mathrm{psi}\left(\mathrm{r}^{2}=0.9968\right)$.

Table 1: Bulk modulus for each gel.

\begin{tabular}{|c|c|}
\hline Gel & Modulus (psi) \\
\hline A & 4672 \\
\hline B & 3029 \\
\hline C & 265 \\
\hline D & 79.2 \\
\hline
\end{tabular}

Table 1 gives the calculated bulk moduli for the gels as obtained through regression analysis of the four graphs. Gel B has a modulus of $3029 \mathrm{psi}$ (20.8MPa), which fits well with other pub- lished reports [29]. The collodion gels, however, are noticeably more compressible. The precipitation of the cellulose as described earlier no doubt led to weakening of the overall structure of the gel. Future tests should mea-sure other mechanical categories.

\section{Conclusions and Discussion}

In this paper I tested the hypothesis that silica organic polymer hybrids lead to stronger aerogels. By using collodion (nitro cellulose) as an additive, I demonstrated that this claim does not hold true for every instance. While the fracture toughness of the cellulose gel may have been greater than that of the pure silica gel, a mercury porosimeter tells us that the bulk modulus is much lower. Future tests on a wide variety of recipes should be examined to find the perfect balance of mechanical properties.

\section{References}

1. Chris D (2004) Using the right bait to catch a comet. The New York Times.

2. Douglas P (1999) Aerogels: Much ado about nothing. Science Spectra.

3. Jung SB, Sung WP, Yang JK, Hyung HP, Haecheon K, et al. (2004) Application of $\mathrm{SiO}_{2}$ aerogel film for interlayer dielectric on GaAs with a barrier of $\mathrm{Si}_{3} \mathrm{~N}_{4}$. Thin Film Solids 447-448: 580-586.

4. Flinn, Edward D (1999) Frozen smoke to capture star dust. Aerospace America 50

5. Paul HL, Kenneth RD (2003) Comparison of thermal insulation performance of fibrous materials for the advanced space suit. J Biomech Eng 125(5): 639-647.

6. Peter J (2002) The light stuff cabot process allows commercial use of 'aerogels'/thinspace. The Boston Globe.

7. Richard S (2003) Plastics engineering. 59(11): 27.

8. (2003) Super aerogels. 45(9): 48.

9. (1996) Silica aerogels absorb kinetic energy. Advanced Materials and Processes 150(6): 5.

10. Milstein M (1999) Bring me the tail of wild-2. Air \& Space Smithsonian 13(6): 54-62.

11. Premachandra JK, Kumudinie C, Mark JE, Dang TD, Arnold FE, et al. (1999) Preparation and properties of some hybrid aero- gels from sulfopolybenzobisthiazole silica composite. Journal of Pure and Applied Chemistry 36(1): 73-83. 
12. Rassy EH, Perrard A, Pierre AC (2003) Behavior of silica aerogel networks as highly porous solid solvent media for lipases in a model transesterification reaction. Chembiochem 4(2-3): 203-210.

13. Catherine AM, Michele L. Anderson, Rhonda MS, Celia IM, et al. (1999) Silica sol as a nanoglue: Flexible synthesis of composite aerogels. Science 284: 622-624.

14. (2004) Transparent magnetic material developed for flat screens Advanced Materials \& Processes 11.

15. Piccolini RJ (1999) Aerogels can be useful in structural, acoustic, electronic, and thermal applications. Chemtech 29(6): 64.

16. Powers KW (1998) The development and characterization of sol gel substrates for chemical and optical applications. Dissertation, University of Florida, USA

17. Dingcai W, RuowenFu, Zhang, Dresselhaus MS, Dresselhaus G, et al. (2004) The preparation of carbon aerogels based upon the gelation of resorcinolfurfural in isopropanol with organic base catalyst. Journal of Non-Crystalline Solids 336: 26-31.

18. Tamon H, Kitamura T, Okazaki M (1998) Preparation of silica aeroge from TEOS. J Colloid Interface Sci 197(2): 353-359.

19. Yamashita J, Ojima T, Shioya M, Hatori H, Yamada Y, et al. (2003) Organic and carbon aerogels derived from poly (vinyl chloride). Carbon 41(2): 285-295.

20. Julie H (2003) Aerogels find new life as a strong and light material. Civil Engineering, 38-39.

21. Nicholas L, Leventis CS, Zhang G, Rawashdeh AMM (2002)
Nanoengineering strong silica aerogels. Nano Letters 2(9): 957-960.

22. Robert SW, Drobeka T, Marcus W, Jochen F, Heckla WM, et al. (1998) Determination of elastic properties of single aerogel powder particles with the AFM. Ultramicroscopy 75: 161-169.

23. Flannery CM (2002) Study of porosity and stiffness measurement of nanoporous aerogel films with tide band ultrasonic surface waves. Ultrasonics 40: 237-242.

24. Masayo 0 (2002) Mercury intrusion porosimetry determines pore-size distribution. American Ceramic Society Bul- letin 81(3): 52-56.

25. Majling J, Komarneni S, Fajnor VS (1995) Mercury porosimeter as a means to measure mechanical properties. Journal of Porous Materials 1(1): 91-95.

26. Perin L, Faivre AS, Etienne C, Woignier T (2004) Nanostructural damage associated with isostatic compression of silica aerogels. Journal of NonCrystalline Solids 333: 68-73.

27. Hafidi AA, Woignier T, Phalippou J, Scherer GW (1998) Room temperature densification of aerogel by isostatic compression. Journal of Sol Gel Science and Technology 13(1-3): 365-369.

28. Perin L, Etienne SC, Faivre A, Phalippou J (2003) Sintering of compressed aerogels. Journal of Non-Crystalline Solids 325: 224-229.

29. George SW, Douglas MS, Xiaomei Q, Anderson JM (1995) Compression of aerogels. Journal of Non-Crystalline Solids 186: 316-320.

30. Paul WA. Characterization of materials by mercury intrusion porosimetry. The Micro Report 11(4): 1-4. 